\title{
Pulmonary disease caused by a newly identified mycobacterium: Mycolicibacterium toneyamachuris: a case report
}

Tomoki Kuge ${ }^{1}$, Kiyoharu Fukushima ${ }^{1,2,3^{*}}$ (D, Yuki Matsumoto ${ }^{4}$, Yuko Abe ${ }^{2}$, Eri Akiba ${ }^{1}$, Kako Haduki ${ }^{1}$, Haruko Saito ${ }^{1}$, Tadayoshi Nitta', Akira Kawano ${ }^{1}$, Takahiro Kawasaki ${ }^{1}$, Takanori Matsuki ${ }^{1}$, Hiroyuki Kagawa' ${ }^{1}$, Daisuke Motooka ${ }^{4}$, Kazuyuki Tsujino ${ }^{1}$, Mari Miki ${ }^{1}$, Keisuke Miki ${ }^{1}$, Seigo Kitada ${ }^{5}$, Shota Nakamura ${ }^{4}$, Tetsuya lida ${ }^{4}$ and Hiroshi Kida ${ }^{1}$

\begin{abstract}
Background: Non-tuberculous mycobacterial pulmonary disease (NTM-PD) is becoming a significant health burden. Recent advances in analysis techniques have allowed the accurate identification of previously unknown NTM species. Here, we report a case of NTM-PD caused by a newly identified mycobacteria in an immunocompetent patient.

Case presentation: A 44-year-old woman was referred to our hospital due to the frequent aggravation of her chronic respiratory symptoms, with NTM-PD-compatible computed tomography findings. Unidentified mycobacterium was repeatedly isolated from respiratory specimens and we diagnosed her as NTM-PD of unidentified mycobacterium. Subsequent whole-genome analysis revealed that the unidentified mycobacterium was a novel mycobacterium genetically close to Mycolicibacterium mucogenicum. We started combination therapy with clarithromycin, moxifloxacin, amikacin, and imipenem/cilastatin, referring to drug sensitivity test results and observed its effect on M. mucogenicum infection. Her symptoms and radiological findings improved significantly.

Conclusion: We report a case of NTM-PD caused by a newly identified mycobacteria, Mycolicibacterium toneyamachuris, genetically close to M. mucogenicum. This pathogenic mycobacterium showed different characteristics from M. mucogenicum about clinical presentation and drug sensitivity. The clinical application of genomic sequencing will advance the identification and classification of pathogenic NTM species, and enhance our understanding of mycobacterial diseases.
\end{abstract}

Keywords: Non-tuberculous mycobacteria, Mycolicibacterium toneyamachuris, Mycolicibacterium mucogenicum, Rapid growing mycobacteria

\footnotetext{
* Correspondence: fukushima@imed3.med.osaka-u.ac.jp

'Department of Respiratory Medicine, National Hospital Organization Osaka

Toneyama Medical Center, 5-1-1 Toneyama Toyonaka, Osaka, Japan

${ }^{2}$ Department of Respiratory Medicine and Clinical Immunology, Osaka

University Graduate School of Medicine, 2-2 Yamadaoka, Suita, Osaka, Japan

Full list of author information is available at the end of the article
}

(C) The Author(s). 2020 Open Access This article is licensed under a Creative Commons Attribution 4.0 International License, which permits use, sharing, adaptation, distribution and reproduction in any medium or format, as long as you give appropriate credit to the original author(s) and the source, provide a link to the Creative Commons licence, and indicate if changes were made. The images or other third party material in this article are included in the article's Creative Commons licence, unless indicated otherwise in a credit line to the material. If material is not included in the article's Creative Commons licence and your intended use is not permitted by statutory regulation or exceeds the permitted use, you will need to obtain permission directly from the copyright holder. To view a copy of this licence, visit http://creativecommons.org/licenses/by/4.0/. The Creative Commons Public Domain Dedication waiver (http://creativecommons.org/publicdomain/zero/1.0/) applies to the data made available in this article, unless otherwise stated in a credit line to the data. 


\section{Background}

The prevalence of non-tuberculous mycobacterial pulmonary disease (NTM-PD) is increasing worldwide and is becoming a significant health burden [1]. Recent advances in analysis techniques have allowed the identification of previously unknown NTM species [2-5].

Here, we report a case of NTM-PD caused by a newly identified mycobacteria genetically close to Mycolicibacterium mucogenicum. This novel mycobacterium caused chronic and progressive pulmonary disease in an immunocompetent patient. Furthermore, drug susceptibility and clinical presentation were different from those reported for M. mucogenicum infections [6].

\section{Case presentation}

A 44-year-old woman, never smoker, was referred to our hospital 18 months ago due to chronic productive cough. She had asthma treated with inhalation therapy and allergic rhinitis. Chest computed tomography showed centrilobular nodules and bronchiectasis in the middle lobe and in the bilateral lower lobes (Fig. 1). Despite treatment with erythromycin and expectorants, her chronic respiratory symptoms worsened. Subsequently, a rapid growing mycobacterium (RGM), strain TY81, was cultured repeatedly from her sputum; however, its species/ subspecies could not be identified by conventional methods such as AccuProbe (Gen-Probe Inc., San Diego, CA, USA), COBAS AMPLICOR (Roche Diagnostic, Tokyo, Japan), or DNA-DNA hybridization assay (Kyokuto Pharmaceutical Industrial, Tokyo, Japan). Therefore, we diagnosed her as NTM-PD of unidentifiable mycobacteria in accordance with the American Thoracic Society/Infectious Diseases Society of America (ATS/ IDSA) criteria for the diagnosis of NTM-PD [7]. Multilocus sequence typing [8] revealed that the unidentified mycobacterium was genetically close to $M$. mucogenicum (Fig. 2).

We performed a whole-genome analysis of TY81 and obtained the complete genome sequence (AP023362AP023365). The DNA G $+C$ content of the type strain was $67.18 \mathrm{~mol} \%$. The mean nucleotide identity (ANI) to M. mucogenicum was $93.3 \%$ and was the maximum value obtained among the type strains of 175 NTM species (Table 1). Phylogenetic analysis using the 16S rRNA sequence suggested that TY81 was closely related to $M$. mucogenicum and related strains. The TY81 strain satisfied three of four conserved signature indels of Mycolicibacterium [9]. The three conserved signatures were a 5 aa insertion of GDAQS at positions 197-201 in the LacI family transcriptional regulator gene, a 1 aa insertion of proline at position 60 in the CDP-diacylglycerolglycerol-3-phosphate 3-phosphatidyl transferase gene, and 1 aa deletion at position 128 in the CDPdiacylglycerol-serine O-phosphatidyl transferase gene. The protein of Cyclase gene (Accession number; WP 066808156) was not detected by homology search using protein-protein Basic Local Alignment Search Tool (BLASTp) with the threshold of $90 \%$ of similarity.

Moreover, we performed additional examination, concerning 16S rRNA phylogeny may not distinguish between closely related species [10]. A comparison was made among TY81, the six strains of M. тисоgenicum and the type strains of $M$. phocaicum and $M$. aubagnense by calculating the ANI values and constructing the phylogenetic tree based on core genomes consisting of 455 genes (Supplementary Fig. 1). The results from both analyses were consistent that all strains belonging to $M$. mucogenicum group are distinct from TY81. Note that the 4 of 6 strains of $M$. mucogenicum were closer to $M$. phocaicum as seen in Behra et al. ${ }^{10}$. Considering the genetic characteristics described above, we suggest that the TY81 strain is a novel species. The scientific name proposed for this species is Mycolicibacterium toneyamachuris sp. nov. with RIMD $1333001^{\mathrm{T}}$ as the type strain. The bacterium was named 'Mycobacterium toneyamachuris' after the place where it was discovered.

$M$. toneyamachuris is a gram-positive, acid-fast, aerobic, anaerobic, non-pigmented and non-motile bacillus. Colonies were grown on 2\% Ogawa-medium, Tryptic Soy Agar (TSA), and 5\% Sheep Blood Agar and appeared greyish without pigmentation (Supplementary Fig. 2). Growth was observed within 7 days at 25,30 , and $37^{\circ} \mathrm{C}$ temperature with optimal growth at $37^{\circ} \mathrm{C}$.

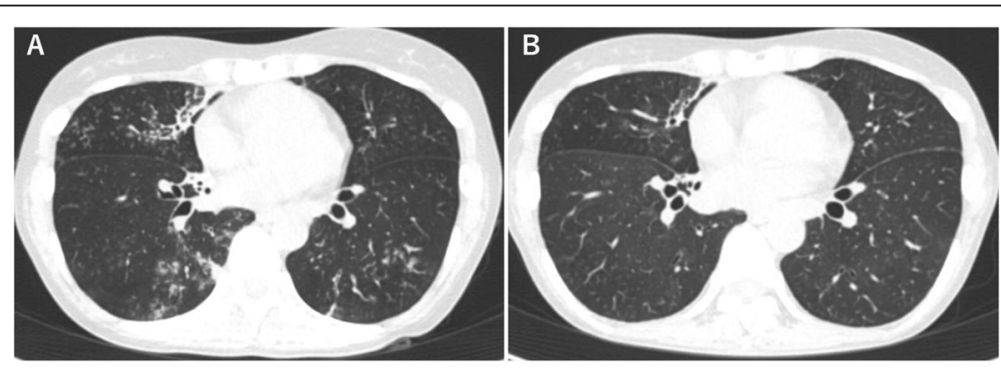

Fig. 1 Chest CT before treatment shows small centrilobular nodules in the middle and lower lobes and slight bronchiectasis with a consolidation in the middle lobe (a). After 2 months treatment, small centrilobular nodules almost vanished (b) 


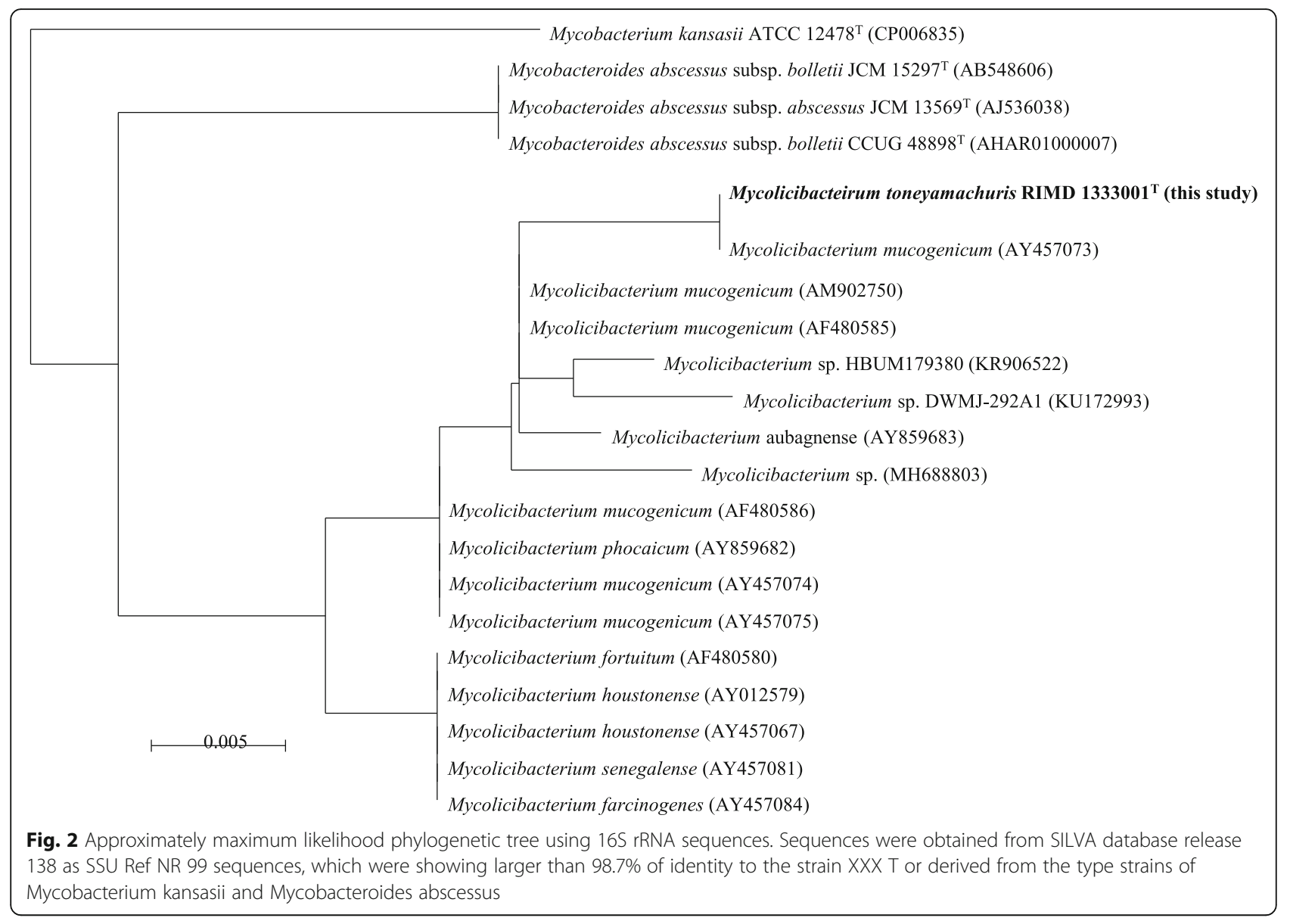

Currently, the treatment of NTM-PD caused by rare species/subspecies is a process of trial and error. It often starts with the drug regimen clinically used for closely related species/subspecies, which is modified by in vitro drug sensitivity test results (Table 2). Because the closest species/subspecies to our strain was $M$. mucogenicum, an RGM for which macrolides, quinolones, and amikacin are the most commonly used drugs [6], we started combination therapy with clarithromycin (CAM) $800 \mathrm{mg} /$ day, moxifloxacin (MFLX) $400 \mathrm{mg} /$ day, amikacin (AMK) $400 \mathrm{mg} /$ day, and imipenem/cilastatin (IPM/CS) 1500 $\mathrm{mg} /$ day. Her clinical symptoms and chest computed tomography 2 months after starting chemotherapy showed significant improvement (Fig. 1).

\section{Discussion and conclusion}

This novel mycobacterium has two different characteristics from the closest species [6]. First, M. тисоgenicum tended to cause catheter-related bacteremia but little pulmonary disease. M. mucogenicum pulmonary disease are reported to be rare and mainly occurs in immunocompromised patients. The patient was immunocompetent. However, we could not exclude the possibilities that treatment with inhaled

Table 1 ANI calculation

\begin{tabular}{llll}
\hline Species name & Strain & Refseq accession number & Refseq category \\
\hline Mycolicibacterium mucogenicum & CSUR P2099 & GCF_001291445.1 & Representative genome \\
Mycolicibacterium phocaicum & JCM 15301 & GCF_010731115.1 & Representative genome \\
Mycolicibacterium aubagnense & JCM 15296 & GCF_3 & Representative genome \\
Mycolicibacterium houstonense & ATCC 49403 & GCF_900078665.2 & Representative genome \\
Mycolicibacterium senegalense & CK2 & GCF_001021425.1 & None \\
Mycobacterium fortuitum & CT6 & GCF_001307545.1 & Representative genome \\
Mycobacteroides abscessus & ATCC 19977 & GCF_000069185.1 & Reference genome \\
\hline
\end{tabular}


Table 2 Antimicrobial drug susceptibility for Mycobacterium toneyamachuris

\begin{tabular}{lll}
\hline Drug & Susceptibility & MIC $(\boldsymbol{\mu} \mathbf{g} / \mathbf{m l})$ \\
\hline CAM (3 days) & S & 0.5 \\
CAM & & 0.5 \\
AZM (3 days) & & 2 \\
AZM & S & 4 \\
CFX & S & 16 \\
IPM & S & 1 \\
MEPM & & 4 \\
FRPM & $\mathrm{S}$ & 8 \\
AMK & $\mathrm{R}$ & 4 \\
TOB & $\mathrm{R}$ & 16 \\
MINO & $\mathrm{R}$ & $>16$ \\
DOXY & $\mathrm{S}$ & $>16$ \\
LZD & $\mathrm{I}$ & $\leq 4$ \\
MFLX & $\mathrm{R}$ & 2 \\
CPFX & & 16 \\
LVFX & $\mathrm{S}$ & 4 \\
ST & $\mathrm{S}$ & $\leq 2 / 38$ \\
\hline SSUSCep & $\mathrm{S}$ &
\end{tabular}

$S$ Susceptible, I Intermediate, $R$ Resistant

corticosteroid made her susceptible to the NTM-PD [11]. Second, our strain showed different drug susceptibility to tetracyclines and quinolones compared with $M$. mucogenicum, an RGM that is relatively susceptible to multidrug treatment. In general, M. mucogenicum is susceptible for amikacin, cefoxitin, clarithromycin, carbapenems, fluoroquinolones and tetracyclines, although, tetracycline resistant straines are detected in about 20 to $40 \%$ of patients. Our strain showed resistance to minocycline, doxycycline and ciprofloxacin.

NTM include mycobacteria other than $M$. tuberculosis and M. leprae and consist of approximately 200 NTM species that are potentially pathogenic $[12,13]$. Because conventional methods have only identified a small number of NTM species, NTM cultured from respiratory samples are sometimes unidentifiable. However, we should strive to identify pathogenic NTM, since NTM have various pathogenicity and prognosis at species/subspecies level $[1,14,15]$. Actually, $M$. toneyamachuris have distinct characteristics from even the closest species; M. mucogenicum. Although one strain does not necessarily represent characteristic of its species, our case indicates importance of accurate identification and usefulness of genomic sequencing.

With the advancement of identification techniques, increasing numbers of novel bacterial species that are potentially pathogenic will be identified in human samples. The identification and classification of undiscovered pathogenic NTM species may enhance our understanding of mycobacterial diseases. In the future, data-sets of clinical phenotypes and bacterial DNA sequences might help elucidate the pathogenesis of NTM.

\section{Supplementary Information}

The online version contains supplementary material available at https://doi. org/10.1186/s12879-020-05626-y.

Additional file 1 Supplementary Figure 1. Whole genomic comparison of $\mathrm{M}$. toneyamachurisand $\mathrm{M}$. mucogenicumgroup. A) Mutual similarity using average nucleotide identity. B) Phylogenetic tree generated by core genome consisting of 455 genes. Supplementary Figure 2. The colony formation of TY81 on Tryptic Soy Agar at $30^{\circ} \mathrm{C}$ at day 7 . Scale bar indicates $2 \mathrm{~mm}$.

\begin{abstract}
Abbreviations
AMK: Amikacin; ANI: Average nucleotide identity; ATS/IDSA: American Thoracic Society/Infectious Diseases Society of America; BLASTp: Proteinprotein Basic Local Alignment Search Tool; CAM: Clarithromycin; CT: Computed tomography; DDH: DNA - DNA hybridization assay; IPM/ CS: Imipenem/cilastatin; MFLX: Moxifloxacin; M. leprae: Mycobacterium lepraeTSA: Tryptic Soy AgarM. mucogenicum: Mycolicibacterium mucogenicum; M. toneyamachuris: Mycobacterium toneyamachuris; $M$. tuberculosis: Mycobacterium tuberculosis; NTM: Non-tuberculous mycobacterial; NTM-PD: Non-tuberculous mycobacterial pulmonary disease; RGM: Rapid growing mycobacterium
\end{abstract}

\section{Acknowledgments}

We thank J. Ludovic Croxford, PhD, from Edanz Group (https://en-authorservices.edanzgroup.com/ac) for editing a draft of this manuscript.

\section{Authors' contributions}

KF designed the project. KF and TK conducted clinical and laboratory data extraction and analysis. YA, EA, KH, HS, TN, AK, TK, TM, HK, KT, MM, KM, SK and $\mathrm{HK}$ assisted with data extraction and analysis. YO, KT, KY, KM, AH and $Y T$ assisted with data analysis. YM, DM and SN performed multi locus typing analysis and whole-genome analysis. TI performed phenotypic

characterization of the culture isolate. KF wrote the manuscript. The authors have read and approved the manuscript.

\section{Funding}

This work was supported by AMED (Grant Number JP20fk0108129). The funder had no role in the design of the study; in the collection, analyses, or interpretation of data; in the writing of the manuscript, or in the decision to publish the results.

\section{Availability of data and materials}

The datasets supporting the conclusions of this article are included within the article.

\section{Ethics approval and consent to participate}

This study was approved by the institutional research ethics board, with a waived requirement for informed consent due to the retrospective nature of the analysis.

\section{Consent for publication}

Written permission for publication of patient information was obtained from the patient presented in this manuscript.

\section{Competing interests}

The authors declare no conflicts of interest to declare. Readers are welcome to comment on the online version of the paper. Correspondence and requests for materials should be addressed to KF. (fukushima@imed3.med. osaka-u.ac.jp). 


\section{Author details}

'Department of Respiratory Medicine, National Hospital Organization Osaka Toneyama Medical Center, 5-1-1 Toneyama Toyonaka, Osaka, Japan. ${ }^{2}$ Department of Respiratory Medicine and Clinical Immunology, Osaka University Graduate School of Medicine, 2-2 Yamadaoka, Suita, Osaka, Japan. ${ }^{3}$ Laboratory of Host Defense, World Premier Institute Immunology Frontier Research Center (WPIIIFReC), Osaka University, 3-1 Yamadaoka, Suita, Osaka, Japan. ${ }^{4}$ Department of Infection Metagenomics, Genome Information Research Center, Research Institute for Microbial Diseases (RIMD), Osaka University, 3-1 Yamadaoka, Suita, Osaka, Japan. ${ }^{5}$ Department of Respiratory Medicine, Yao Tokushukai General Hospital, 1-17 Wakakusa-cho, Yao, Osaka, Japan

Received: 23 August 2020 Accepted: 17 November 2020

Published online: 25 November 2020

\section{References}

1. Daley CL, laccarino JM, Lange C, Cambau E, Wallace RJ, Andrejak C, et al. Treatment of Nontuberculous mycobacterial pulmonary disease: an official ATS/ERS/ESCMID/IDSA clinical practice guideline: executive summary. Clin Infect Dis. 2020. https://doi.org/10.1093/cid/ciaa241.

2. Yoshida M, Fukano H, Ogura Y, Kazumi Y, Mitarai S, Hayashi T, et al. Complete genome sequence of mycobacterium shigaense. Genome Announc. 2018;6:e00552-18.

3. Fukano H, Hiranuma O, Matsui Y, Tanaka S, Hoshino $Y$. The first case of chronic pulmonary mycobacterium shigaense infection in an immunocompetent patient. New Microbes New Infect. 2020;33:100630.

4. Saito H, Iwamoto T, Ohkusu K, Otsuka Y, Akiyama Y, Sato S, et al. Mycobacterium shinjukuense sp. nov., a slowly growing, non-chromogenic species isolated from human clinical specimens. Int J Syst Evol Microbiol. 2011;61:1927-32.

5. Takeda K, Ohshima N, Nagai H, Sato R, Ando T, Kusaka K, et al. Six cases of pulmonary mycobacterium shinjukuense infection at a single hospital. Intern Med. 2016;55:787-91.

6. Adekambi T. MycobacteriuM. Mucogenicum group infections: a review. Clin Microbiol Infect. 2009;15:911-8.

7. Griffith DE, Aksamit T, Brown-Elliott BA, Catanzaro A, Daley C, Gordin F, et al. An official ATS/IDSA statement: diagnosis, treatment, and prevention of nontuberculous mycobacterial diseases. Am J Respir Crit Care Med. 2007; 175:367-416.

8. Matsumoto Y, Kinjo T, Motooka D, Nabeya D, Jung N, Uechi K, et al. Comprehensive subspecies identification of 175 nontuberculous mycobacteria species based on 7547 genomic profiles. Emerg Microbes Infect. 2019;8:1043-53.

9. Gupta RS, Lo B, Son J. Phylogenomics and comparative genomic studies robustly support division of the genus mycobacterium into an emended genus mycobacterium and four novel genera. Front Microbiol. 2018;9:67.

10. Behra PRK, Pettersson BMF, Das S, Dasgupta S, Kirsebom LA. Comparative genomics of mycobacterium mucogenicum and mycobacterium neoaurum clade members emphasizing tRNA and non-coding RNA. BMC Evol Biol. 2019;19:124.

11. Brode SK, Campitelli MA, Kwong JC, Lu H, Marchand-Austin A, Gershon AS, et al. The risk of mycobacterial infections associated with inhaled corticosteroid use. Eur Respir J. 2017;50:1700037.

12. Fedrizzi T, Meehan CJ, Grottola A, Giacobazzi E, Fregni Serpini G, Tagliazucchi S, et al. Genomic characterization of Nontuberculous mycobacteria. Sci Rep. 2017;7:45258.

13. Philley JV, Griffith DE. Medical Management of Pulmonary Nontuberculous Mycobacterial Disease. Thorac Surg Clin. 2019;29:65-76.

14. Boyle DP, Zembower TR, Reddy S, Qi C. Comparison of clinical features, virulence, and relapse among Mycobacterium avium complex species. Am J Respir Crit Care Med. 2015;191:1310-7.

15. Kwak N, Dalcolmo MP, Daley CL, Eather G, Gayoso R, Hasegawa N, et al. Mycobacterium abscessus pulmonary disease: individual patient data metaanalysis. Eur Respir J. 2019;54:1801991.

\section{Publisher's Note}

Springer Nature remains neutral with regard to jurisdictional claims in published maps and institutional affiliations.

\section{Ready to submit your research? Choose BMC and benefit from:}

- fast, convenient online submission

- thorough peer review by experienced researchers in your field

- rapid publication on acceptance

- support for research data, including large and complex data types

- gold Open Access which fosters wider collaboration and increased citations

- maximum visibility for your research: over $100 \mathrm{M}$ website views per year

At BMC, research is always in progress.

Learn more biomedcentral.com/submissions 